\title{
CULTURA Y SOCIEDAD
}

\author{
El problema mente-cuerpo en la \\ antigua China: discusiones recientes
}

\author{
The mind-body problem \\ in Ancient China: Recent discussions
}

FEDERICO DANIEL MINA*

Resumen: Si bien el problema mente-cuerpo ha sido central en la tradición del pensamiento indoeuropeo, los filósofos de la antigua China mostraron escaso interés en el tema. A pesar de ello, la posición que implícitamente defendieron ha sido muy discutida en tiempos recientes. Un consenso entre los historiadores es que nunca respaldaron un dualismo ontológico fuerte y que, de hecho, esa idea habría sido extraña en China antes de la introducción del budismo. Los supuestos ontológicos e intelectuales de la época parecen contribuir a esa lectura. Sin embargo, en los últimos años algunos autores han argumentado que sí había un dualismo mente-cuerpo en ese periodo, pero mi análisis crítico de estas posiciones concluye que sus argumentos son insuficientes para refutar el consenso tradicional.

Palabras clave: filosofía china; China antigua; problema mentecuerpo; alma; corazón.

Abstract: Even though the mind-body problem has been a primary concern in Indo-European thought, philosophers in Ancient China

Recepción: 23 de enero de 2018. / Aceptación: 2 de mayo de 2018.

* Universidad Nacional de Córdoba, fede_mina@hotmail.com 
showed little interest in the subject. However, in recent times, the implicit defense of their position has been fiercely debated. Historians have formed a consensus that they never supported a strong ontological dualism and that furthermore such an idea would have been a strange in pre-Buddhist China. The ontological and intellectual assumptions of the time support this view. Some scholars have recently argued evidence exists of mind-body dualism at that time but my critical analysis of these proposals concludes that their arguments are not strong enough to refute the traditional consensus.

Key words: Chinese philosophy; Ancient China; mind-body problem; soul; heart.

El problema mente-cuerpo ha sido central en la filosofía indoeuropea. En comparación, la filosofía pre-Qin en China sorprendentemente casi no exhibe interés explícito en el tema. Pese a que uno de los focos de discusión fue la naturaleza humana, y en particular qué hace a los humanos diferentes del resto de los seres, el lugar donde residen las facultades específicas de los hombres no recibió demasiada atención de los pensadores del periodo. El término que suele traducirse como mente es 心 (xin), que etimológicamente se refiere sólo al corazón. Sin embargo, no hay unanimidad sobre las funciones específicas que corresponden al xin y, por tanto, sobre hasta qué punto es válido traducirlo como "mente" o como "corazón”. Además, la religión y la cosmología no incorporan seres o realidades trascendentes, sino más bien lo contrario, tanto los principios de la naturaleza como las actividades de los espíritus y los dioses parecen estar íntimamente ligados al mundo social. Por ello los historiadores del pensamiento en ese periodo suelen sostener que la idea de que la mente es una entidad ontológicamente diferente al resto del cuerpo es ajena al pensamiento chino preimperial, e incluso hasta la introducción del budismo en el país. En los últimos años, sin embargo, algunos autores han puesto en duda esta lectura.

Comenzaré presentando los términos que fueron usados para referirse al alma o espíritu con el fin de analizar su impacto en el pensamiento filosófico. Luego expondré algunas de las posiciones tradicionales que se resisten a considerar que haya 
habido un dualismo ontológico en la antigua China, para analizar, entonces, tres desafíos recientes a esas posturas. En primer lugar, Paul Goldin, quien ha aseverado que en las prácticas religiosas hay evidencia de que sí había un dualismo ontológico fuerte; ${ }^{1}$ Xunwu Chen, por su parte, afirma que en Mencio la mente es una sustancia racional diferente del cuerpo; $y$, por último, Edward Slingerland sostiene en una serie de trabajos que no hay certeza de un dualismo ontológico cartesiano, pero sí de un dualismo más débil. Para defender esto, recurre a un análisis cuantitativo de la bibliografía pre-Qin, lo cual constituye un enfoque prometedor en las discusiones del tema. Un análisis crítico de estas tres perspectivas muestra, sin embargo, que tienen dificultades internas o requieren de más argumentos para refutar la posición tradicional.

\section{Términos para "espíritu" en la religión y la filosofía tempranas de China}

Desde la filosofía griega -en especial en el Fedón de Platón y el De Anima de Aristóteles- y el cristianismo, la capacidad intelectiva del hombre se asoció con la posesión de un alma. El paradigma moderno de dualismo en Occidente ha sido el cartesianismo, que postula que esa capacidad no está radicada en un cuerpo (el cerebro, el sistema nervioso o algo similar), sino en un tipo diferente de sustancia sin extensión espacial e independiente de los procesos y las leyes de la naturaleza que también identifica con el alma. Si bien no es necesario que el dualismo adopte conceptos de origen religioso, con el fin de ser exhaustivo comenzaré con un análisis del concepto de "alma" o "espíritu” en China.

La existencia de un "alma" o "espíritu" no era extraña a las prácticas religiosas chinas y, por lo tanto, no era desconocida por Confucio. Ciertamente, tampoco por su gran rival, Mozi, quien cita historias e incluso defiende que los fantasmas exis-

${ }^{1}$ Con este término refiero a un dualismo de sustancias, a veces mal llamado dualismo cartesiano, en su versión más radical, es decir, la creencia de que la actividad mental se desarrolla en un tipo de entidad con atributos esenciales diferentes y antagónicos con el tipo de entidad que tienen los objetos físicos. 
ten, aunque no hay consenso entre los historiadores sobre la posición que le atribuye una genuina creencia en fantasmas y la que postula que su defensa es sólo pragmática, para evitar que las personas incurran en malas acciones cuando creen que nadie los observa. Pese a esto, ninguno de los filósofos posteriores durante el periodo de los Reinos Combatientes intentó definir con precisión las características distintivas del "alma" ni contrastar sus funciones específicas con las del cuerpo.

Comenzaré con un breve repaso de los tres términos más relevantes para discutir las ideas de alma en las prácticas religiosas de la antigua China. El primero es el genérico shen (神), cuyo significado principal es espíritu o fantasma. Hay registros desde la dinastía Shang y proviene de creencias anteriores al inicio de la escritura china. Los rituales orientados a los espíritus de los antepasados están fuertemente arraigados a esta cultura.

Más problemáticos son los otros dos términos: bun (魂) y po (魄), que remiten, respectivamente, a un alma sutil relacionada con el cielo y la energía yang, y a una versión más terrenal del alma relacionada con el cuerpo, la tierra y la energía yin. A diferencia de shen, que era usado para referir tanto a los espíritus de los muertos como a una parte integrante de las personas, bun y po solían usarse sólo en referencia a los seres vivos. Puede verse que los dos caracteres comparten el componente semántico de “fantasma” (鬼), pero difieren en el radical. El de po (el más antiguo de los dos términos) va acompañado de "blanco", con la connotación de brillante, y el de bun (probablemente creado en contraposición a po), de "nube", que en el contexto puede interpretarse como falta de luz. De acuerdo con Schuessler (2007, pp. 290, 417), esto quizá se deba a la concepción de que el alma bun podría permanecer de algún modo luego de la muerte. Sin embargo, esas definiciones y distinciones entre los términos no pueden ser atribuidas al tiempo de Confucio. De acuerdo con Yü (1987, pp. 371, 374), apenas en la dinastía Han maduró con claridad esa concepción de las dos almas, y hacia finales del siglo vi a.e.c., el concepto de bun seguía siendo relativamente nuevo. En uno de los artículos que analizaré más adelante, Slingerland (2013, p. 30) llega a una conclusión parecida: "a medida que avanza 
el [periodo de] los Reinos Combatientes, el espíritu [shen] es discutido junto con al menos otros dos tipos subordinados de almas, po 魄 y bun 魂”. ${ }^{2}$ Es decir, la distinción de las dos almas fue casi nula en el periodo de Primavera y Otoño, se consolidó gradualmente durante los Reinos Combatientes y maduró en la dinastía Han. Conforme la separación fue creciendo, los papeles también cambiaron. De este modo, a po se le fueron asignando funciones más bien corporales y nutritivas (vagamente similar al "alma vegetativa" de Aristóteles) y a hun, las capacidades cognitivas, un asiento en el xin (corazón/mente) y cierta permanencia aun después de la muerte de la persona. No hay evidencia de que ninguna de ellas haya sido concebida como entidad inmaterial, más bien ambas estaban constituidas de qi. Tampoco la inmaterialidad es una característica del alma. ${ }^{3}$ Por lo tanto, no hay evidencia de que un dualismo ontológico fuerte subyazga a estas creencias religiosas; luego analizaremos una posición contraria a esta afirmación.

El uso de estos términos en el contexto filosófico es bastante escaso: la mención de las dos almas es virtualmente inexistente entre los pensadores pre-Qin. Ninguno de los dos aparece en el Lunyu de Confucio, ni en Mozi ni en Mencio. En el Dao De Jing sí se usa po en el primer verso del capítulo $10 .{ }^{4}$ Algunos traductores consideran que ese verso debe entenderse como una contraposición entre dos versiones de almas (Ames y Hall, 2010), pero el verso contrasta po y ying (營), y ellos igualan éste a bun, lo cual es discutible. Dejando la cuestión de lado, es interesante notar que este capítulo no aparece en la versión del texto más antigua disponible, la de las excavaciones en Guodian descubierta en 1993 (Perkins, 2014, p. 32). Por supuesto, no hay evidencia concluyente para afirmar que esta versión sea la original y que los pasajes que no aparecen allí sean interpolaciones posteriores, pero puede funcionar como hipótesis de trabajo hasta que tengamos mayor conocimiento de la evolu-

2 Salvo indicación contraria, todas las traducciones son mías.

${ }^{3}$ Dice Yü (1987, p. 379): "la idea de que el alma individual puede sobrevivir indefinidamente luego de la muerte parece extraña a la mentalidad china”.

${ }^{4}$ En el comentario de Heshang Gong (dinastía Han) al Dao De Jing, en la explicación de este pasaje y otros se usan los términos bun y po, lo cual corrobora la afirmación anterior de que, en esa dinastía, ya estaba más establecida la definición de ese tipo de almas. 
ción del texto. De cualquier modo, el pasaje sigue y vincula las almas mencionadas con el $q i$, por lo que se descarta una concepción completamente trascendental del alma en Laozi. En el Xunzi sí están ambos términos. En el capítulo 14, bun asoma como una cita rimada, pero Hutton (2014, n. 4, p. 142) afirma que puede ser una interpolación al texto original, ya que el pasaje contradice la concepción del cielo del autor y no parece tener mucho sentido en el contexto en que aparece. En el capítulo 22 del libro se menciona po, pero con un sentido diferente al que analizamos.

En cuanto a shen, sí está presente en los primeros textos filosóficos; sin embargo, con cierta reticencia (con la posible excepción de Mozi, ya mencionado). En Confucio, la pregunta por los espíritus es caracterizada como superflua: la preocupación por los problemas del hombre es más importante que los debates sobre los fantasmas (Analectas, 11.12). Mencio parece haber adoptado la sugerencia de su maestro intelectual, ya que alude a shen cuando describe rituales, por lo que el foco no es la existencia misma de los espíritus, sino la correcta ejecución de los rituales establecidos (5A5 y 7A13). En el supuesto de que la versión del Dao De Jing encontrada en Guodian sea el estrato más antiguo del texto, el término no aparece; pero sí está en los capítulos 9, 29, 39 y 60 de la versión recibida de 81 capítulos. ${ }^{5}$ También está presente en el Taiyi Sheng Shui, que posiblemente sea uno de los primeros textos filosóficos en desarrollar una cosmología y una cosmogonía en China, pero como shenming (神明) (Galvany, 2003). De acuerdo con Yongyun Lee (2010), los dos caracteres no deben interpretarse como una palabra (lo cual es un uso de fines del periodo de los Reinos Combatientes), ya que aparecen como un elemento más en la sucesión de procesos de generación cósmica, rodeados por fases compuestas cada una por un conjunto de elementos simétricos y opuestos (cielo y tierra, yin y yang, cuatro estaciones, frío y calor, etc.). De hecho, puede verse en esa sucesión de fases que ninguno de los elementos tiene una dimensión espiritual ni una capacidad cognitiva. Esto es congruente con la posición daoísta

${ }^{5}$ Esta afirmación apoyaría la tesis general de que la preocupación por las almas fue creciendo en la dinastía Han. Si bien es especulativa, podría ayudar a comprender la evolución del daoísmo hacia su vertiente religiosa. 
de que la creación no involucra elementos volitivos, sino que es espontánea (ziran 自然). Por lo tanto, la significación religiosa de shen se naturaliza al incluirse en la cosmología.

\section{El corazón-mente (心) en el pensamiento antiguo}

Pasemos ahora de la discusión de los espíritus y las almas al problema específicamente filosófico de qué es la mente. Como ya dije, es difícil encontrar referencias explícitas a la pregunta ontológica sobre la mente, y es necesario reconstruir con cuidado las posiciones sobre las diferencias en funcionamiento que la mente tiene respecto a los órganos del cuerpo y los tres términos de origen religioso ya señalados. También debemos evitar que nuestros prejuicios culturales nos hagan adjudicar enfoques tradicionales indoeuropeos al pensamiento chino antiguo. Ciertamente, la traducción a lenguas occidentales propicia esos prejuicios. Por ejemplo, hemos usado el término "religión" sin discriminar las particularidades chinas (véase Schwartz, 1985, pp. $19-20$ y ss.). Podemos traducir xin como "mente", pero hecha la salvedad de que, al menos etimológicamente, se refiere a un órgano corporal: el corazón. Asimismo, se puede traducir shen como "espíritu" o "alma", ya que en la religión china suele remitir a aquello que permanece luego de la muerte del cuerpo, pero es incorrecto suponer que el espíritu es idéntico a la mente o que es inmortal. Por ello es necesario tener en cuenta aspectos generales y cuestiones específicas respecto a la mente del pensamiento chino.

La principal dificultad para atribuir un dualismo ontológico fuerte a los pensadores del periodo pre-Qin es el consenso generalizado de que, en el periodo previo al contacto con el budismo, los chinos no postularon realidades trascendentales; más bien, todas sus teorías se basan en un continuo entre cielo y tierra, los objetos y lo indiferenciado, la energía positiva y la negativa, etc. Hay una continua transformación de la realidad de la que ningún elemento escapa. Es ajena por completo la idea de un Dios absolutamente trascendente o de un mundo de las ideas que posea todos los atributos de perfección en contraste con el mundo visible. Incluso los mandatos del cielo 
(tianming) están interpenetrados con la realidad social y natural de tal manera que es imposible pensar que su origen sea un plano separado del mundo. Esa concepción holista atribuye al qi el sustrato común a todas las cosas, de acuerdo con Liu (2015, p. 39): "en la visión china de mundo [...] todas las dimensiones de lo que hay pueden ubicarse en un espectro continuo de operaciones del qi”. El dualismo ontológico implicaría, contrariamente, que o bien hay cosas en el mundo que no están compuestas de qi o que hay al menos dos tipos de qi suficientemente diferenciados como para considerarlos sustancias diferentes (lo cual traería la dificultad de explicar por qué se continuó usando el mismo término para referirse a ambas).

Más allá de estas consideraciones, la historiografía se ha opuesto tajantemente a la posibilidad de que hubiera un dualismo fuerte en la época. Herbert Fingarette, en su libro Confucius: The secular as sacred, fue uno de los primeros en insistir en el anacronismo de utilizar un lenguaje referido a la vida mental para interpretar a Confucio. Según dice, los conceptos psicológicos de sentimientos, actitudes e ideas internas son parte de la tradición budista y occidental (1998, p. 37). Confucio sólo piensa en términos de acción y circunstancias visibles al público, no acerca de estados subjetivos (p. 40). Las personas sólo reaccionan a realidades externas, por lo que los términos traducidos como sentimientos son conceptos funcionales que causan y generan comportamientos públicos. Según afirma, Confucio nunca tuvo ninguna noción de una vida mental interna, ni siquiera es una posibilidad que haya rechazado (p. 45). Si bien su análisis es específicamente sobre Confucio, sus conclusiones se extienden a otros pensadores de su tiempo. Aunque en el libro nunca discute ontológicamente el problema de la mente, su posición es la crítica más radical a la posibilidad de que haya habido un dualismo en la antigua China. Al no haber nada en el discurso filosófico que se refiera a una psicología interna y a considerar que los procesos cognitivos y emocionales no tienen otra dimensión más que la pública, la pregunta misma por la mente -entendida como el lugar donde se radican esos procesos- carece de sentido. La única conclusión de estas ideas no sólo es que no hay una entidad incorpórea donde radiquen sentimientos y razonamientos, sino 
que ninguna entidad (el corazón, xin) puede identificarse con el concepto psicologista de mente que aparece en la tradición indoeuropea. De hecho, en el libro no hay una discusión sobre el término xin (que seguramente traduciría como "corazón”) y el significado y las funciones que le adscribe.

Algunas objeciones se han hecho contra esta interpretación, al señalar que se asemeja a una posición conductista de las personas en la que sólo hay una respuesta al medio (Rosemont, 1978; Liu y Shun, 1996). Reilly (2012) recientemente criticó esas objeciones en un intento por rescatar los aspectos positivos de su obra (que sin duda son muchos). Destaco su afirmación de que, para Fingarette (1998, p. 69), la ejecución de actos rituales no es resultado de una mera coerción, sino más bien algo análogo a la virtuosa ejecución de una obra musical que no puede "actuarse" sino que requiere "actos sutiles e inteligentes que exhiben más o menos sensibilidad al contexto” (p. 53). A pesar de usar términos como "sensibilidad" e "inteligencia", no es necesario asumir una vida interna, porque la ejecución es un acto público. Reilly argumenta, entonces, que el ser individual no puede reducirse a meras respuestas al medio, sino que tiene una dignidad inherente. Esa discusión no es importante para los propósitos de este trabajo; las interpretaciones de Fingarette son congruentes con la ya provista: no hay funciones mentales específicas y, por lo tanto, la pregunta por la mente en tiempos de Confucio no tiene sentido. La "sensibilidad" y la "inteligencia" pueden atribuirse a una persona en tanto ejecute su papel social, pero no a una parte específica de esa persona.

Si bien la posición de Fingarette es demasiado radical al rechazar toda dimensión individual de la actividad, varios historiadores han aceptado sus objeciones al lenguaje mentalista mencionado anteriormente. Por ejemplo, Angus Graham (2013, p. 25) también dice del dualismo mente-cuerpo que “nunca surgió en la filosofía pre-Han; la palabra bsin [xin] 心 'corazón' es a veces traducida como 'mente', lo cual es razonable para la filosofía posterior influida por el budismo, pero en el periodo clásico sólo se refiere al corazón en tanto órgano con el cual uno piensa, aprueba o desaprueba”. Más aún, Graham (pp. 95-105) específicamente defiende que la distinción interno-externo, referida al sujeto, emerge apenas en el siglo IV 
a.e.c. con Song Xing y el autor del anónimo Neiye. Sin embargo, esta diferenciación no implica un dualismo ontológico, sino que se usa para distinguir sentimientos naturalmente presentes en la persona (y, por lo tanto, relativamente libres de influencias externas), o para referirse al estado del qi interno del sujeto.

Siguiendo esta línea, Chad Hansen (1992, p. 15) también caracteriza el dualismo como una teoría extraña a los pensadores chinos del periodo, y explica que sólo logró desarrollarse cuando el budismo entró en el reino. Incluso afirma que el daoísmo antiguo fue más propenso a ser interpretado con base en estas ideas foráneas debido a la posición neoconfuciana de que daoísmo y budismo son similares. Las distinciones comunes entre razón y emoción o conciencia privada y vida pública no son aplicables al pensamiento chino antiguo; son categorías impuestas con un modelo mente-cuerpo que no existía en ese momento. Más aún, la idea de una sustancia mental privada no tiene sentido debido al continuo que hay entre la vida social y el individuo. En el caso particular de Mencio, incluso afirma que la moralidad está anclada tanto en el corazón-mente del hombre como en el qi, lo que diluye la dicotomía entre sujeto interno y mundo externo. Hansen (1992, pp. 75, 84 y ss.) sí es más claro al remarcar la característica distintiva del corazónmente como órgano del cuerpo capaz de juzgar.

Harold Roth (1990) llega a similares conclusiones al analizar el concepto de shen en textos daoístas de la época. De acuerdo con sus argumentos, para entender los procesos psicológicos y cognitivos debemos considerar la profunda interpenetración del qi con todo el cuerpo, y repasa varios fragmentos en los que se menciona la importancia de cultivar el cuerpo como prerrequisito para alcanzar una comprensión intelectual. Respecto al debate mente-cuerpo, concluye: "Más que un fantasma en una máquina, lo que hallamos aquí es una posición en la que fantasma y máquina se han fusionado en un todo íntegro y que sólo puede ser separado en la mente analítica del que lo observa” (p. 26). Thomas Michael (2005, p. 101), también refiriéndose a textos daoístas, considera que shen se puede traducir como "mente" o "espíritu”, pero "se refiere a un estado de energía corporal muy refinado" dentro de un continuo de sustancias en el que jing (精, remite a líquidos corporales) es la parte 
más densa del cuerpo, qi una intermedia y shen la más refinada. Se podría objetar que los textos considerados en estos dos trabajos no cubren todas las tradiciones de pensamiento de la antigüedad, lo cual, si bien es cierto, es una instancia más en contra de la idea de que es posible sostener una clara distinción entre las facultades cognitivas del xin y el resto del cuerpo. Es más, ambos textos clasifican el Neiye como texto daoísta y, con base en él, argumentan sus posiciones, pero algunos autores afirman que, de hecho, pueden encontrarse evidentes similitudes entre éste y Mencio (por ejemplo, Graham, 2013, p. 105.)

\section{Shen como mente incorpórea}

En un artículo reciente, Paul Goldin (2015) intenta refutar la posición clásica al recuperar textos religiosos sobre los espíritus con el objetivo de mostrar que la distinción mente-cuerpo es la que mejor explica el contenido de esos textos. La mayoría de lo que traduce indica estados psicológicos de los espíritus en ceremonias religiosas. En el pasaje más extenso, que él considera que proporciona la imagen más clara de un dualismo, refiere a una ceremonia en la que una persona es usada como receptáculo para que los espíritus de los antepasados hablen a través de ella. El pasaje concluye con la frase: "iLos espíritus están borrachos!”. Goldin entonces dice:

No creo que sea posible comprender un poema como el Jizui sin abandonar la apresurada afirmación de Graham de que en la filosofía prebudista nunca hubo una dicotomía mente-cuerpo. ¿Qué otra cosa son los espíritus ancestrales que poseen un cuerpo si no mentes incorpóreas haciendo uso temporal de un cuerpo externo? (p. 79).

$\mathrm{Ni}$ ese pasaje ni los anteriores permiten, sin embargo, arribar a esa conclusión. No hay duda de que las versiones más extremas de la posición historiográfica tradicional, aquellas en que se afirma que no había siquiera una noción de vida interna, son muy difíciles de sostener en vista de estas ceremonias. Pero otras posibilidades ontológicas son compatibles con los fragmentos de rituales que Goldin utiliza. El concepto de "mente incorpórea" ("disembodied mind" en el original) no es- 
tá justificado en ninguno de esos pasajes. En su brevísima conclusión menciona, sin desarrollar un argumento, que la existencia de los espíritus y los fantasmas "no es material, ya que no puede ser percibida del mismo modo que percibimos las cosas materiales" (p. 87). Esto por mencionar que no eran visibles, pero sus posiciones son problemáticas y merecen más atención.

En primer lugar - como muestra Goldin-, evidentemente los espíritus tienen las funciones que atribuimos a la mente, pero en ningún lado se indica que no estén constituidos del mismo tipo de materia que el cuerpo ni que el espíritu sólo retenga las funciones de la mente (en cuyo caso sería sólo la mente la que sobrevive a la muerte). De hecho, los mismos pasajes citados por Goldin muestran que los espíritus consumen comida y bebida (aunque la parte más sutil de ambas, por lo cual no es visible su consumo). Eso implica que el espíritu no sólo tiene las funciones que atribuimos a la mente, sino también las nutritivas que son propias del cuerpo. Por otro lado, el pasaje que habla de los espíritus borrachos sugiere que las funciones mentales se ven afectadas por el consumo de esas sustancias. Si el alcohol afecta la mente, lo más razonable es suponer que cuerpo y mente están interconectados, que uno puede causar efectos en el otro y viceversa. Entonces, a la dependencia nutritiva que tiene el espíritu, debemos agregar la dependencia causal, dos características que se alejan de la interpretación de un dualismo ontológico. Por último, la rápida advertencia de que los espíritus no son visibles de ninguna manera constituye una prueba de que están compuestos de otra sustancia. Ya mencioné a autores que afirman que en ese periodo la realidad se pensaba como un continuo de $q i$. Una de las acepciones de $q i$ (氣) en esa época es justamente "aire", una sustancia invisible, pero a la que nadie consideraría inmaterial. ${ }^{6}$ No por ello el aire es de una sustancia radicalmente diferente que el resto de las cosas. Con todo esto no estoy dudando de que la postura de Goldin sea una de las maneras posibles de leer los pasajes. Pero no parece

Así, Brian van Norden (2008, p. xxviii) dice que para Mencio y sus contemporáneos, "el qi es un tipo de fluido presente tanto en la atmósfera como en el cuerpo humano y capaz de responder al tipo y la intensidad de las reacciones emocionales de la persona”. 
justificado el salto de un dualismo débil (es decir, la mente tiene funciones que le son propias y que no tienen otras sustancias materiales) a un dualismo ontológico como el que defiende.

\section{Xin como sustancia distinta al cuerpo en Mencio}

Otro de los autores que cree que había un dualismo fuerte es Xunwu Chen. Cuando discute sobre Mencio, le atribuye cuatro tesis:

i) la mente humana es una sustancia mental distintiva; ii) todas las mentes humanas originariamente contienen una mente moral que consiste en las facultades de empatía, vergüenza y repugnancia, y respeto, así como en la capacidad de distinguir entre el bien y el mal; iii) todas las mentes humanas están constituidas originariamente para tener benevolencia, rectitud, corrección y sabiduría, y iv) todas las mentes humanas originariamente tienen una naturaleza racional universal (Chen, 2016, p. 4).

La segunda y la cuarta tesis son afirmaciones cercanas al original y tienen una base textual sólida, aunque puede discutirse la traducción de algunos de esos términos a lenguas occidentales; pero sí son cuestionables las otras dos. Comienzo con la primera afirmación, de la cual no hay duda que remite a un dualismo ontológico, que luego expande Chen cuando sostiene que "la mente humana es una sustancia mental dicotómica del cuerpo" (p. 4). Gran parte de su argumento se basa en la lectura de Mencio 6B15, quien afirma que el cielo (tian 天) sometió a situaciones adversas tanto el cuerpo como la mente de varios grandes sabios de la antigüedad. El pasaje, en general, parece indicar que la mente depende fuertemente de la situación del cuerpo, aunque no es la lectura que Chen hace. Según apunta, el pasaje opone la mente a la "entidad de la piel” (término con que traduce tifu 體膚), algo que no parece tener sustento en el texto. ${ }^{7}$ Además, afirma que en ese pasaje se

${ }^{7}$ El fragmento al que refiere dice: “必先苦其心志, 勞其筋骨, 餓其體膚, 空乏其 身”; una lectura alternativa del pasaje podría ser que mente y voluntad (心志), músculos y huesos (筋骨) y “entidad de la piel” son partes del mismo cuerpo (身), donde la última oración es una conclusión general de las oraciones anteriores. 
contrasta el entrenamiento de la mente con el entrenamiento del cuerpo, y dado que Mencio considera que lo relativo a la mente es más importante que lo relativo al cuerpo, concluye que las actividades mentales son irreductibles a las del cuerpo. Esto más bien sería un argumento a favor de un dualismo de funciones, no ontológico. Una lectura más razonable del pasaje sería que, cuando Mencio enumera las características distintivas de la mente, no lo hace porque suponga que constituye una sustancia ontológica diferente, sino para remarcar el potencial de los humanos para elaborar una capacidad moral superior a las posibilidades del cuerpo y que nos hace superiores a los animales. Las funciones morales no contradicen la visión tradicional de que el universo es concebido como un continuo de $q i$ con diferentes niveles de condensación. Para jerarquizar la capacidad moral del hombre no es necesario postular que está radicada en una sustancia diferente al resto del cuerpo, sino más bien que fue impuesta por el cielo a los hombres, como proponen Liu y Shun (1996).

Por otro lado, Chen (2016) sostiene que las mentes tienen una naturaleza "racional". Se basa en un pasaje del capítulo Gaozi I (6A7) en el que se dice: “¿en qué, pues, somos iguales en lo que tiene que ver con el corazón (心)?: en lo que llamamos principios naturales [ $l i$ 理] y en la rectitud [ $y i$ 義]” (Pérez Arroyo, 2002, p. 334; la interposición de caracteres chinos en la traducción es mía). Chen (2016, p. 4) afirma que tanto li como yi sólo pueden ser predicados de la sustancia mental, lo cual la hace cualitativamente diferente de la material. El problema es que traduce $l i$ como "racional", cuando nada lo justifica. En cambio, considero preferible que se traduzca como "principio" (teniendo en cuenta que es un significado distinto del que luego darían al término los neoconfucianos) u "orden", como lo hace Van Norden (2008, p. 151), ya que "racional" evoca un concepto de razón en la tradición occidental que no tiene paralelo entre los pensadores chinos del periodo. ${ }^{8}$ Además, Chen hace una afirmación demasiado controversial con base en

${ }^{8}$ En Occidente, la razón es usualmente contrapuesta a los sentimientos. En China, en cambio, hay una correlación positiva entre sabiduría (智) y ciertas cualidades morales que involucran la capacidad de empatía con otras personas, en particular ren (仁). 
un solo pasaje. El término $l i$ aparece en otros dos pasajes del Mencio: en 5B10 es usado para referirse al sonido armonioso de los instrumentos musicales, por lo tanto, en el sentido de "orden", y en 7B19, li, de acuerdo con van Norden, debe traducirse como "articulado", es decir, también en el sentido de “orden”. En vista de estos ejemplos en el mismo libro, es difícil sostener que $l i$ sólo puede ser atribuido a la sustancia mental, salvo el improbable caso (que igualmente Chen no justifica) de que en ese pasaje su uso sea diferente al del resto. Además, el término no aparece en las Analectas, por lo que Mencio en ese pasaje debería haber inventado un nuevo sentido para el término dentro de la tradición confuciana.

La razón por la que creo que Chen incurre en estos errores es porque adopta una postura historiográfica demasiado clásica: analizar a los primeros pensadores confucianos desde la perspectiva de los filósofos neoconfucianos de las dinastías Song a Ming (algo que también hace en Chen, 2017). Eso tiene al menos dos consecuencias que claramente aparecen en sus trabajos. Por un lado, las posiciones de Confucio son forzadas a armonizar con las de Mencio y separadas de las de Xunzi. Por otro lado, se evalúa el confucianismo antiguo con base en los conceptos del neoconfucianismo. El objetivo central de los dos trabajos mencionados de Chen es mostrar que, entre los pensadores de ambos grupos, hay posiciones similares respecto a la mente, en particular la consideración de que es una sustancia diferente del cuerpo. Mientras que esa afirmación puede ser poco problemática para el neoconfucianismo (que pudo ser influido por el budismo), es muy equívoca para la filosofía pre-Qin. Una argumentación más extensa en contra de esta posición historiográfica en general se puede ver en Hansen (1992, pp. 5-18).

\section{El aporte del análisis cuantitativo a la discusión}

El tercer desafío a la posición tradicional surgió en una serie de artículos de Edward Slingerland y otros (2011, 2013, 2017). A diferencia de Goldin y Chen, no intentan sostener un dualismo ontológico, sino un "dualismo débil”, donde el xin es la sede de 
las funciones más importantes y distintivas de los hombres y que crecientemente fue contrastándose con el resto de los órganos, lo cual le otorgaría un estatus especial respecto a éstos. La originalidad de su trabajo radica en el uso de métodos cuantitativos de análisis textual para comprobar su hipótesis, lo cual sigue siendo relativamente rechazado por muchos investigadores en las humanidades. Sin embargo, considero que los resultados que ha obtenido hasta ahora son prometedores para continuar indagando.

En su artículo de 2013 utilizó bases de datos con textos pre-Qin y extrajo pasajes que contuvieran el término xin. Después obtuvo un muestreo aleatorio de 60 pasajes que fue analizado y clasificado cualitativamente de acuerdo con 29 categorías desarrolladas inductivamente en las que examinó la relación que xin tenía con el cuerpo o con órganos particulares (si era implícita o explícitamente contrastado con el cuerpo, si no era clara una jerarquía entre ambos, si había una relación con términos vinculados a emociones, etc.). Luego los pasajes fueron clasificados dependiendo del periodo histórico al que pertenecían en relación con los Reinos Combatientes: anteriores (desde Dinastía Shang hasta 475 a.e.c.), principios (fines del siglo $\mathrm{v}$ hasta mediados del siglo IV a.e.c.) y finales (mediados del siglo Iv a 221 a.e.c.). El resultado más relevante de este análisis surgió al compararse la evolución de las definiciones del xin como centro de funciones cognitivas, emocionales o como un mero órgano. La frecuencia de esta última concepción es casi nula en los tres periodos. En el previo a los Estados Combatientes, las funciones cognitivas y las emocionales tenían relativamente una frecuencia similar. Sin embargo, durante las dos divisiones mencionadas de los Reinos Combatientes, claramente esas funciones son diferenciadas: $80 \%$ de los pasajes con funciones cognitivas asociadas al xin en comparación con $10 \%$ al que se le asocian funciones emocionales. Esos datos sugieren que, durante esa etapa, el xin era considerado el centro de las habilidades más distintivas de las personas: planificación, uso del lenguaje, volición, etc. (2013, p. 20).

No entraré en los detalles técnicos metodológicos del trabajo de Slingerland, Nichols, Neilbo y Logan (2017). Aquí utilizan métodos más complejos (mutual information, hierarchical 
clustering y probabilidad bayesiana) para analizar la frecuencia con la que cada par de términos de una lista preestablecida ocurre en corpus de textos pre-Qin, y obtienen patrones de la manera en que aparecen. En particular comparan la coocurrencia del término xin y el de otros órganos con los tres vocablos que se refieren al cuerpo en la China antigua: shen (身), $t i$ (體) y xing (形). Los resultados fueron que los órganos internos del cuerpo con frecuencia aparecen relacionados entre sí, mientras que xin suele mostrarse en conjunción con los órganos asociados a la comunicación y la percepción (boca, ojo y oído), y en especial contrastado con términos del cuerpo. Estos resultados refuerzan los del artículo anterior: el xin es una parte especial del humano claramente diferenciable de otras partes del cuerpo y más cercano a nuestro concepto de "mente" que al de "corazón" al que originariamente remitía.

Algunos de los enfoques y los resultados de Slingerland han sido cuestionados. En Klein y Klein (2012) se enumeran tres razones para dudar de algunas de sus conclusiones (en particular, las de Slingerland y Chudek, 2011). Dos de ellas conciernen a la periodización usada. Por un lado, argumentan que el Clásico de las odas (Shijing 詩經), en el periodo anterior a los Reinos Combatientes, distorsiona los resultados por su excesivo peso estadístico (constituye 38\% de los datos de ese periodo). Obviamente, la escasez de material de este periodo es una de las causas, pero arguyen que, al tratarse de una obra integrada por canciones, himnos y poesía, las correlaciones entre corazón y emociones son más altas que en otras épocas. Eso explicaría por qué en los resultados de aquella investigación (2011), los datos correspondientes al periodo anterior a los Reinos Combatientes se apartan de la norma. De hecho, Klein y Klein repiten la cuantificación eliminando el Clásico de las odas y el resultado es que, durante los tres periodos marcados por Slingerland, la asociación de emociones y xin, por un lado, y facultades cognitivas y xin, por el otro, se mantiene relativamente estable. El otro problema sobre la periodización es más importante aún, y se refiere a la escasez de información confiable que permita datar los textos para clasificarlos como pertenecientes a un periodo u otro. La historiografía actual suele concordar en que prácticamente todos los textos de la 
época en realidad son recopilaciones de fragmentos de distintos periodos. Sólo por mencionar unos ejemplos, los últimos cinco capítulos del Lunyu han sido considerados apéndices posteriores al menos desde la dinastía Qing (Makenhan, 1996, pp. 9-10); en la dinastía Han, el Mengzi contenía cuatro capítulos hoy perdidos (Van Norden, 2007, p. 212), y Franklin Perkins (2014) sugiere que los capítulos 67 a 81 del Dao De Jing pueden no sólo ser un añadido posterior al texto original, sino que incluso es posible que hayan conformado un texto separado, debido a la coherencia interna que presentan. Además, es ampliamente reconocido que los capítulos finales (que constituyen cuantitativamente más de la mitad de las obras) del Mozi y del Zhuangzi son escritos posteriores a los primeros capítulos.

En su respuesta a estas objeciones, Slingerland y Chudek (2012) sostienen que el Clásico de las odas es una obra heterogénea compuesta tanto por textos líricos como por otros en los que las emociones no necesariamente están exaltadas. También argumentan que, dada la escasez de textos en ese primer periodo, no considerar esa obra implicaría contar con muy pocos datos para sacar conclusiones. Respecto a la otra objeción afirman que si bien las dudas textuales sobre casi todas las obras ciertamente están presentes, hay razones "filológicas y filosóficas" para asumir que la periodización es correcta en general, al menos en esos tres amplios periodos. Lamentablemente, no expanden su argumento en este punto, pero parecería indicar una circularidad. Cuando defienden la introducción de métodos cuantitativos en el análisis de las humanidades, arguyen que tienen el potencial de poner en duda nuestros supuestos y nuestros prejuicios. Sin embargo, es necesario algo más que un supuesto sobre las razones para considerar correcta la periodicidad si es que los resultados van a ser útiles para cambiar nuestros supuestos filológicos (por ejemplo, en qué periodos debemos traducir xin como "corazónmente" y en cuáles sólo como "mente") y filosóficos (puede o no sostenerse un dualismo débil en el periodo). Justamente en sus trabajos podrían evaluar diferentes resultados dependiendo del supuesto que se asuma. Por ejemplo, podrían considerar, cómo en el trabajo de Klein y Klein, los resultados con o sin el Clásico de las odas. 
Además, hay otro problema con el tipo de supuestos que asumen. Es posible que la versión de los textos que ha llegado a nuestros días refleje correctamente las ideas de los pensadores antiguos, pero sabemos que el trabajo de los copistas posteriores alteró la redacción de algunos textos, por ejemplo, con el remplazo de ciertos caracteres por sinónimos, con las restricciones sobre el tabú de algunos caracteres, etc. Dado que su investigación cuantifica la ocurrencia de ciertos caracteres, esos cambios relativamente menores del original pueden tener un impacto importante en los resultados finales. Esto sucede porque la gramática y el contenido de ciertos textos indicarían su clasificación en un periodo anterior a aquel en que se efectuaron esos cambios menores de redacción, pero los resultados estarían sesgados hacia este último periodo. Por ejemplo, respecto al Clásico de documentos (Shang Shu 尚書), Sarah Allan (2012, p. 548) descree de un análisis cuantitativo, ya que "aun si esos capítulos son auténticos, han sido copiados por un periodo de más de 3000 años, especialmente a la escritura de la dinastía Han, por lo que cierta corrupción es inevitable”. Esto tiene especial relevancia en el caso de ese libro, ya que junto al Clásico de las odas es uno de los textos con más influencia en los resultados del periodo anterior a los Reinos Combatientes, pero de cuyo original no tenemos ninguna copia. Ahora bien, si en el análisis de ese periodo no se toman en cuenta estos dos clásicos, la cantidad de información disponible resulta estadísticamente insignificante. Esto no invalida la investigación, pero sí anima a tener cautela con sus resultados.

A diferencia de los autores analizados antes, Slingerland utiliza estos análisis cuantitativos para atribuir al pensamiento pre-Qin un dualismo "débil", como lo llama, es decir una posición en la que la mente y el cuerpo tienen funciones y actividades diferentes, pero que muchas veces se solapan entre sí. De hecho, parte de las motivaciones que guían los trabajos mencionados es poner a prueba las hipótesis de que el dualismo está presente en todas las tradiciones religiosas y culturales del mundo (Slingerland y Chudek, 2011, pp. 997-999; Slingerland et al., 2017, pp. 2, 24). 


\section{Reflexiones finales}

En este trabajo se analizaron tres desafíos a la posición tradicional respecto a la mente y el cuerpo en la China pre-Qin. Primero debemos notar que la divergencia de interpretaciones es posible debido a una indeterminación real en los textos. Además, estamos partiendo de una pregunta que en la reflexión indoeuropea fue muy importante, pero que carecía de interés en aquel otro contexto. Los filósofos chinos del periodo no reflexionaron extensamente sobre el contraste entre las funciones cognitivas y emocionales y los procesos fisiológicos de los órganos del cuerpo. De ninguna manera puede tomarse esto como un indicio de que hubo una falta de perspicacia o creatividad. Por el contrario, nos indica que si bien algunos problemas filosóficos parecen ser universales (como el gobierno ideal, qué es la virtud, etc.), responden a las circunstancias culturales y sociales específicas en que surgen. Sucede lo mismo en el ámbito religioso con problemas como el lugar de Dios o de los dioses, el alma o la vida eterna. Esto no significa que no debamos preguntarnos por la concepción implícita en estos ámbitos. El estudio de estos supuestos teóricos puede ayudarnos a entender otras características del pensamiento de cada autor y también ser útil en la producción de filosofía comparativa entre China y Occidente, lo cual, a su vez, redundará en un mejor entendimiento de nuestras tradiciones intelectuales.

Sin embargo, hablar de la indeterminación de los textos implica que admiten varias lecturas, pero no que todas sean válidas. El enfoque tradicional afirma que en el pensamiento antiguo chino no hay sustancias ontológicamente opuestas e irreductibles entre sí. Esa idea es congruente con las cosmologías de las diferentes escuelas. Pero también hay enfoques que niegan toda posibilidad de separar la mente del cuerpo, incluso en términos de funciones específicas, como es el caso de Fingarette en su análisis de Confucio. Llamaremos a la primera la versión moderada de la posición tradicional, y a la última, la versión radical.

La versión radical no tiene mucho sustento y carece de defensores actualmente. Tanto el análisis de textos religiosos de Gol- 
din como las investigaciones de Slingerland realmente muestran que, a finales del periodo de Primavera y Otoño, el corazónmente era generalmente reconocido como el lugar donde se asentaban las funciones cognitivas y emocionales de la persona. La sugerencia de Fingarette de que una vida mental interna o de que las funciones mentales específicas del ser humano no estaban presentes en el pensamiento de Confucio ni siquiera como posibilidad rechazada es, por tanto, poco plausible. También la idea de que se pueda explicar el confucianismo (al menos antes de Mencio) sin mencionar las emociones. Fingarette, como varios han notado, evita analizar conceptos clave que aparecen en las Analectas, como lo correcto ( $y i$ 義), o deja sin explicar por qué si los conceptos que indican cómo actúa una persona sólo se refieren a la dimensión pública y manifiesta de la acción, varios de los caracteres que lo componen contienen el radical de corazón-mente (Fu, 1978).

La versión moderada, en cambio, fue y es sostenida por muchos autores. Independientemente de cuáles sean las funciones específicas del corazón-mente, sostienen que no es razonable considerarlo una sustancia ontológica diferente del resto del cuerpo. En los trabajos de Chen vimos que una lectura bastante libre de ciertos conceptos en la obra de Mencio conduce a una visión dualista de ellos, pero sin una base textual que apoye esa conclusión.

Goldin cree que es posible defender un dualismo fuerte a partir de la lectura de cómo ciertos rituales religiosos parecen referirse a un espíritu de un antepasado que toma posesión del cuerpo de un vivo para comunicarse a través de él. El evento parecería probar la posición cartesiana clásica: el cuerpo es una especie de maquinaria sin pensamiento hasta que un alma toma control de él. Pero hemos visto la debilidad de sus argumentos, pues los mismos pasajes que usa para defender esa postura pueden interpretarse con la idea de que la mente es sólo una versión rarificada del mismo qi que compone el cuerpo. Además, aun si su interpretación fuera la que mejor explica los textos religiosos que analiza, no puede transferirse esa conclusión basada en rituales religiosos a los filósofos del periodo. Como hemos visto, desde un comienzo mostraron cierta indiferencia o escepticismo hacia los espí- 
ritus o los fantasmas en las discusiones sobre la naturaleza humana. ${ }^{9}$

Las investigaciones de Slingerland son bastante prometedoras porque incluyen nuevos métodos en la discusión de las humanidades. Sin embargo, también el impacto en la discusión sobre los pensadores en particular resulta dudoso. Si bien sus conclusiones muestran tendencias generales sobre el uso de ciertos términos, aún resta el trabajo de analizar cómo se reflejaron en cada autor en particular. Por ejemplo, si aceptamos los tres periodos en los que divide la literatura pre-Qin para analizar la relación del corazón-mente con ciertas funciones cognitivas, tendríamos que dilucidar si Confucio fue un representante del primer periodo o del segundo. Además, las objeciones metodológicas planteadas por Klein y Klein ciertamente contribuyen a la discusión sobre la utilidad de los métodos cuantitativos en las humanidades.

En conclusión, hay un mayor número de estudios que afirman, cada vez con más énfasis, que en la antigua China el corazón-mente era considerado un órgano especial en tanto se le atribuían ciertas funciones específicas. Pero aquellos que aseguran, con base en lo anterior, que esa especificidad era tan marcada que permite suponer un dualismo ontológico fuerte en el periodo, aún parecen insuficientes.

Dirección institucional del autor:

Facultad de Filosofía y Humanidades

Universidad Nacional de Córdoba

Haya de la Torre s/ $n$, Ciudad Universitaria

5000 Córdoba, Argentina

\section{Referencias}

Allan, S. (2012). On Shu 書 (Documents) and the origin of the Shang shu 尚書 (Ancient Documents) in light of recently discovered

\footnotetext{
${ }^{9}$ Michael Puett (2002) rescata el interés sobre las funciones y las capacidades de los espíritus que comienza a crecer en algunos textos y en prácticas chamánicas, especialmente desde el siglo Iv a.e.c. y a comienzos del periodo imperial. Agradezco a un evaluador(a) anónimo(a) por recordar este punto.
} 
bamboo slip manuscripts. Bulletin of the School of Oriental and African Studies, 75(3), 547-557. https://dx.doi.org/10.1017/ S0041977X12000547

Ames, R. y Hall, D. (Trads.). (2010). Dao de Jing 'Making this life significant': A philosophical translation. Nueva York, NY: Ballantine Books.

Chen, X. (2016). The problem of mind in Confucianism. Asian Philosophy, 26(2), 166-181. https://dx.doi.org/10.1080/095523 67.2016.1165790

Chen, X. (2017). Mind and space: A Confucian perspective. Asian Philosophy, 27(1), 1-15. https://dx.doi.org/10.1080/09552367.2 017.1288694

FingaretTe, H. (1998). Confucius: The secular as sacred, Long Grove, ILL.: Waveland Press.

Fu, C. (1978). Fingarette and Munro on early Confucianism: A methodological examination. Philosophy East and West, 28(2), 181-198. https://dx.doi.org/10.2307/1397742

Galvany, A. (2003). Estudio preliminar de un manuscrito taoísta hallado en China: Tai yi Sheng Shui. Boletín de la Asociación Española de Orientalistas, 39, 257-269.

Goldin, P. (2015). The consciousness of the dead as a philosophical problem in ancient China. En R. A. H. King. (Ed.), The good life and conceptions of life in early China and Groeco-Roman Antiquity (pp. 59-92). Berlín: De Gruyter.

GraHAm, A. C. (2013). El dao en disputa: la argumentación filosófica en la China antigua. México: Fondo de Cultura Económica.

Hansen, C. (1992). A Daoist theory of Chinese thought: A philosophical interpretation. Nueva York: Oxford University Press.

Hutton, E. L. (Trad.). (2014). Xunzi: The complete text. Princeton, NJ: Princeton University Press.

KleIN, E. y Klein, C. (2012). Did the Chinese have a change of heart? Cognitive Science, 36(2), 179-182. https://dx.doi.org/10.1111/ j.1551-6709.2011.01231.x

LEE, Y.-Y. (2010). A naturalistic understanding of Taiyi Sheng Shui cosmology. Recuperado de http://cccp.uchicago.edu/ archive/2010Creel-LucePaleographyWorkshop/Yong-yun\%20 Lee\%20-\%20on\%20Taiyi\%20sheng\%20shui\%20Cosmology. pdf

LiU, J. L. (2015). In defense of Chineseqi-naturalism. En C. Li y F. Perkins (Eds.), Chinese metaphysics and its problems (pp. 3353). Cambridge: Cambridge University Press. https://dx.doi. org/10.1017/CBO9781316145180.003 
Liu, S. y Shun, K. (1996). Some reflections on Mencius' views of mind-heart and human nature. Philosophy East and West, 46(2), 143-164. https://dx.doi.org/10.2307/1399402

Makeham, J. (1996). The formation of Lunyu as a book. Monumenta Serica, 44(1), 1-24.

Michael, T. (2005). The Pristine Dao: Metaphysics in early Daoist discourse. (Ed. R. T. Ames). Albany, NY: State University of New York Press.

Pérez Arroyo, J. (Trad.). (2002). Los cuatro libros. Barcelona: Paidós. Perkins, F. (2014). Divergences within the Lăož́: A study of chapters 67-81. T'oung Pao, 100(1-3), 1-32. https://dx.doi. org/10.1163/15685322-10013p01

PuetT, M. J. (2002). To become a God: Cosmology, sacrifice, and selfdivinization in early China. Cambridge, MA: Harvard University Asia Center for the Harvard-Yenching Institute.

ReILly, R. (2012). Fingarette on moral agency in the Analects. Philosophy East and West, 62(4), 529-544. https://dx.doi.org/10.1353/ pew.2012.0057

Rosemont, H. Jr. (1978). Reply to Professor Fingarette. Philosophy East and West, 28(4), 515-519. https://dx.doi.org/10.2307/1398654

Roth, H. D. (1990). The early Taoist concept of Shen: A ghost in the machine? En K. Smith (Ed.), Sagehood and systematizing thought in warring states and Han China (pp. 11-32). Brunswick, ME: Bowdoin College.

Schuessler, A. (2007). ABC etymological dictionary of old Chinese. Honolulu, HI: University of Hawai'i Press.

Schwartz, B. (1985). The world of thought in ancient China. Cambridge, MA: Belknap Press.

StingerLAND, E. (2013). Body and mind in early China: An integrated humanities-science approach. Journal of the American Academy of Religion, 81(1), 6-55. https://dx.doi.org/10.1093/jaarel/lfs094

SLINGERLAND, E. y Chudek, M. (2011). The prevalence of mindbody dualism in early China. Cognitive Science, 35(5), 997-1007. https://dx.doi.org/10.1111/j.1551-6709.2011.01186.x

SLINGERLAND, E. y Chudek, M. (2012). The challenges of qualitatively coding ancient texts. Cognitive Science, 36(2), 183-186. https:// dx.doi.org/10.1111/j.1551-6709.2011.01232.x

Slingerland, E., Nichols, R., Neilbo, K. y Logan, C. (2017). The distant reading of religious texts: A "big data" approach to mindbody concepts in early China. Journal of the American Academy of Religion, 85(4), 985-1016. https://dx.doi.org/10.1093/jaarel/ lfw090 
Van Norden, B. (2007). Virtue ethics and consequentialism in early Chinese philosophy. Cambridge: Cambridge University Press.

Van Norden, B. (2008). Mengzi: With selections from traditional commentaries. Indianapolis, IN: Hackett Publishing.

YÜ, Y. (1987). “O soul, come back!” A study in the changing conceptions of the soul and afterlife in pre-Buddhist China. Harvard Journal of Asiatic Studies, 47(2), 363-395. https://dx.doi. org/10.2307/2719187 
